Archives de sciences sociales des religions

131-132 | juillet - décembre 2005

Varia

\title{
Genèse des émotions au sein des Assemblées de Dieu polynésiennes
}

Yannick Fer

\section{(2) OpenEdition}

12 Journals

Édition électronique

URL : http://journals.openedition.org/assr/3265

DOI : 10.4000/assr.3265

ISSN : $1777-5825$

Éditeur

Éditions de l'EHESS

Édition imprimée

Date de publication : 1 décembre 2005

Pagination : 143-163

ISBN : 2-7132-2045-9

ISSN : 0335-5985

\section{Référence électronique}

Yannick Fer, "Genèse des émotions au sein des Assemblées de Dieu polynésiennes », Archives de sciences sociales des religions [En ligne], 131-132 | juillet - décembre 2005, mis en ligne le 30 juin 2008 , consulté le 19 avril 2019. URL : http://journals.openedition.org/assr/3265 ; DOI : 10.4000/assr.3265

Ce document a été généré automatiquement le 19 avril 2019

(C) Archives de sciences sociales des religions 


\title{
Genèse des émotions au sein des Assemblées de Dieu polynésiennes
}

\author{
Yannick Fer
}

1 Entamant son "voyage en pays pentecôtiste ", H. Cox établit une distinction liminaire entre les croyances des fondamentalistes protestants, qui «se sont exprimées dans des systèmes théologiques formalisés » et celles des pentecôtistes, davantage "portées par des témoignages, des paroles extatiques et des mouvements corporels ». Mais il précise aussitôt :

Ceci ne veut pas dire pour autant que le pentecôtisme ne véhicule pas un ensemble de vues et d'idées religieuses. Il s'agit bien d'une théologie, de tout un univers religieux, d'un système complexe de symboles qui répondent aux éternelles questions de l'homme sur le sens de la vie et les valeurs ${ }^{1}$.

On ne peut mieux résumer les inclinations ambivalentes que suscite le pentecôtisme chez bien des observateurs: d'abord portés à souligner la faiblesse (ou l'indigence) de ses constructions théologiques pour mieux insister sur l'effervescence émotionnelle qui s'y manifeste, il leur faut également rendre compte des processus de rationalisation des vies personnelles observables dans beaucoup d'églises de sensibilité pentecôtiste. Mais du désordre (des émotions), peut-il émerger une mise en ordre (des vies)? Et si la reconfiguration des conduites personnelles n'est pas imputable aux seuls moments de forte intensité émotionnelle où communient les fidèles, sur quel système normatif, quels dispositifs d'apprentissage et de contrôle repose-t-elle? Autrement dit, comment s'articulent au sein d'un même espace religieux l'instabilité des émotions et la stabilité apportée par une lecture biblique littérale et une moralisation des conduites?

C'est cette difficile conciliation que pointe J.-P. Willaime quand, tout en inscrivant le pentecôtisme dans la logique protestante du sola scriptura, il remarque que «le privilège accordé à l'expérience émotionnelle et à l'efficacité divine hic et nunc, l'insistance sur l'oralité et l'immédiateté peuvent aussi aboutir à une minoration du texte biblique (...) ou à une nouvelle monopolisation cléricale des Écritures reposant sur une division du travail 
religieux : l'effervescence émotionnelle pour les masses, la science des Écritures pour les pasteurs gérant les émotions de leurs ouailles. ${ }^{2}$ "

Indéniablement, cette incertitude est liée à la fois à la diversité considérable des pentecôtismes contemporains et à l'oscillation du modèle pentecôtiste lui-même entre deux modes idéaux-typiques de régulation religieuse : idéologique, avec « une théologie protestante privilégiant une lecture normative et peu contextualisée »; charismatique quand les manifestations spectaculaires de la puissance divine l'emportent sur tout discours doctrinal ${ }^{3}$. Mais elle traduit aussi le poids, dans le champ sociologique, d'une certaine " tradition d'animosité " ${ }^{4}$ à l'égard des émotions - notamment en religion - qui, à travers un jeu d'oppositions centrées sur le couple émotion/raison, s'interdit trop souvent d'aborder la signification d'un certain nombre d'expressions religieuses émotionnelles autrement que sur le registre de la régression.

5 En écartant autant que possible ces "notions anticipées », on s'efforcera donc ici, sur la base d'une enquête de terrain conduite de septembre 2000 à août 2002 au sein des Assemblées de Dieu de Polynésie française ${ }^{5}$, de «se mettre (comme y invitait É. Durkheim) en face des faits eux-mêmes ${ }^{6}{ }^{6}$. Ces faits n'ont pas, à eux seuls, valeur de généralité : les Assemblées de Dieu, majoritaires au sein du pentecôtisme polynésien, ne représentent qu'une partie - la plus ancienne et, souvent, la plus «classique » - des pentecôtismes contemporains; les assemblées polynésiennes se sont développées à la croisée de plusieurs influences culturelles spécifiques: celles de l'Action missionnaire française, de la communauté chinoise hakka de Tahiti (premier terrain d'implantation du pentecôtisme, au début des années 1960), de la culture polynésienne enfin, qui - depuis la fin du XVIII siècle, date des premières missions protestantes européennes - a fait de la Bible le pilier de son identité «traditionnelle » 7 . Pour autant, n'y voir que l'expression d'une irréductible singularité ("exotique ») serait écarter trop hâtivement des faits suffisamment significatifs pour inciter à un réexamen critique du statut de l'émotion en pentecôtisme.

6 Car, au sein des Assemblées de Dieu polynésiennes, l'intensité émotionnelle apparaît moins comme une expérience originaire que l'institution s'emploie à canaliser que comme l'expression en actes d'un ensemble de valeurs, de connaissances et de dispositions inculquées par le biais d'un travail institutionnel « invisible », dont le modus operandi vise à conforter les fidèles dans l'« enchantement » d'une relation personnelle avec Dieu.

7 L'effervescence des émotions et la rationalisation des vies personnelles peuvent alors être ressaisies comme les deux versants d'un ethos pentecôtiste fondé sur un véritable «évangile relationnel ». C'est en effet l'instauration d'une communication constante et transparente avec un Dieu proche qui, d'une part entraîne une stabilisation des vies en soumettant les intentions personnelles, les conflits et les interpellations du monde à cette « médiation divine » et, d'autre part, encourage les fidèles à « se dire »- sans restriction ni formalité - quand dans la prière et (surtout) pendant les cultes, Dieu peut leur répondre « immédiatement ».

8 Si le pentecôtisme a pu être, à bon droit, qualifié de "protestantisme émotionnel », il le doit avant tout à l'effervescence de ses cultes. L'intensité des chants, du flot de paroles déversé pêle-mêle, accompagnées de larmes, de cris, de guérisons miraculeuses et, parfois, de transes, contraste en bien des endroits avec la retenue que les Églises protestantes « historiques » ont associé au sacré. Ainsi, dans les Églises de Polynésie, les missionnaires de la London Missionary Society ont diffusé, à partir de la fin du XVIII ${ }^{e}$ siècle, 
un modèle cultuel empreint d'une stricte solennité, qui visait d'abord à contenir tout débordement de la "nature » polynésienne, mais s'est maintenu jusqu'à aujourd'hui comme l'expression consacrée du respect dû à Dieu et à la mémoire des ancêtres qui, les premiers, ont cru. Dans un tel contexte, les cultes pentecôtistes ont été spontanément perçus par les fidèles du protestantisme traditionnel comme une innovation intrigante et transgressive.

Le contraste est tout aussi net si l'on oppose les formes de religiosité les plus courantes du christianisme européen, marqué par un double processus historique de rationalisation selon l'expression de M. Weber - et d'intériorisation subjective de l'expérience religieuse, aux pratiques collectives de haute intensité émotionnelle observables dans bien des cultures non-occidentales où, précisément, le pentecôtisme rencontre des succès considérables. Il est alors tentant d'y voir, comme le fait J.-P. Bastian, une sorte d'affinité ontologique entre la « simplicité » des croyances pentecôtistes, le caractère apparemment irraisonné des expressions émotionnelles qu'elles encouragent, et des dispositions culturelles supposées communes à un vaste ensemble qu'il situe "aux périphéries de l'Occident $»^{8}$. Cette répartition géographique rudimentaire, qui renvoie davantage au "grand partage" disciplinaire établi au XIX ${ }^{e}$ siècle (et à l'invention des "sociétés primitives » comme champ de l'altérité et domaine de recherche dévolu à l'ethnologie) qu'à une unité culturelle improbable, a cependant le mérite de souligner la dimension éminemment culturelle (et sociale) des modalités d'expression de l'émotion - y compris au sein du pentecôtisme. G. Rivière remarque ainsi, à propos des fidèles aymaras des Églises pentecôtistes boliviennes :

$\mathrm{Si}$, dans les temples de l'IEPB [église évangélique pentecôtiste bolivienne], ruraux et urbains, l'émotion peut s'exprimer avec plus de force et de liberté que dans l'Église catholique par exemple, elle reste contenue. Les fidèles ne cessent d'appartenir à une culture qui condamne les excès et où le contrôle de soi est une qualité appréciée. Et il ajoute : L'apprentissage des formes d'expression de l'émotion est un thème qui reste à défricher 9 .

10 En effet, le pentecôtisme ne semble pas abolir les écarts liés à des économies affectives différenciées, c'est-à-dire, à la fois, les systèmes de significations culturels susceptibles de faire naître des émotions et les capacités des individus à communiquer leurs états émotionnels. Mais l'analyse sociologique du pentecôtisme s'en tient quant à elle, pour l'essentiel, à la recherche des modes d'encadrement et de mise en sens d'émotions envisagées selon un point de vue strictement universaliste: ce qui varie, ce n'est pas le contenu des émotions, mais le niveau et les formes du contrôle exercé sur elles par la raison individuelle, les normes culturelles et l'institution religieuse. L'émotion elle-même reste une sorte de donnée "brute ", toujours identique car renvoyant sans l'ombre d'un doute à une seule origine : l'« émotion des profondeurs ", envisagée " à la fois comme une “expérience" primitive » (c'est-à-dire comme «forme élémentaire » de la religion, d'un point de vue chronologique) et comme une expérience « originaire » (c'est-à-dire comme forme fondatrice du sentiment religieux, d'un point de vue génétique) ${ }^{10}$.

11 C'est à partir des descriptions ethnographiques de cérémonies célébrées périodiquement par les sociétés australiennes, lors de rassemblements succédant à de longues périodes de dispersion, qu'É. Durkheim a mis en évidence cette émotion fondamentale :

Nous avons vu en effet - conclut-il - que si la vie collective, quand elle atteint un certain degré d'intensité, donne l'éveil à la pensée religieuse, c'est parce qu'elle détermine un état d'effervescence qui change les conditions de l'activité psychique. 
Les énergies vitales sont surexcitées, les passions plus vives, les sensations plus

fortes ; il en est même qui ne se produisent qu'à ce moment ${ }^{11}$.

12 Porté par cet enthousiasme collectif, chacun se sent élevé au-dessus de lui-même et « entraîné par une sorte de pouvoir extérieur qui le fait penser et agir autrement qu'en temps normal, il a naturellement l'impression de n'être plus lui-même, (...) tout se passe comme s'il était réellement transporté dans un monde spécial, entièrement différent de celui où il vit d'ordinaire, dans un milieu tout peuplé de forces exceptionnellement intenses, qui l'envahissent et le métamorphosent. " ${ }^{12}$

13 Cette exaltation est-elle un phénomène physique, lié à la coalescence d'individus jusquelà dispersés? Ou l'expression passionnée du sentiment que la société inspire à ses membres quand ils en éprouvent confusément l'existence, au-delà de la simple addition des individus? Ou encore tout cela à la fois ? É. Durkheim s'intéresse d'abord aux effets mécaniques du rassemblement :

Or, note-t-il, le seul fait de l'agglomération agit comme un excitant exceptionnellement puissant. Une fois les individus assemblés il se dégage de leur rapprochement une sorte d'électricité qui les transporte à un degré extraordinaire d'exaltation ${ }^{13}$.

14 Mais cette excitation «électrique » n'est pas une pure force matérielle précédant toute construction sociale, elle naît au contraire du fait que chaque participant se reconnaît comme appartenant à un même ensemble solidaire et retrouve la société dont il s'était éloigné. La figure totémique permettant de matérialiser aux yeux des individus cette permanence de la vie sociale, "la force religieuse " apparaît finalement comme «le sentiment que la collectivité inspire à ses membres, mais projeté hors des consciences qui l'éprouvent, et objectivé. $"{ }^{14}$ Autrement dit, si la profondeur de l'émotion manifestée lors des cérémonies renvoie à un stade élémentaire, ce n'est pas celui où se crée la société, mais celui où l'individu est agi par la société sans être encore capable d'en prendre conscience. Car le " primitif » est, pour É. Durkheim, un être dont « les facultés émotives et passionnelles (...) ne sont qu'imparfaitement soumises au contrôle de sa raison et de sa volonté, il perd aisément la maîtrise de soi. » ${ }^{15} \mathrm{Il}$ " sent qu'il est élevé au-dessus de luimême ", mais son intelligence "rudimentaire " l'empêche de se représenter nettement l'origine de ces sensations extraordinaires ${ }^{16}$. L'émotion religieuse ne précède pas le social, elle l'exprime.

15 Si l'on choisissait de rattacher l'émotion pentecôtiste à cette expérience élémentaire, il faudrait donc la distinguer, a minima, des émotions relevant d'un "palier inférieur ", c'est-à-dire - selon l'approche dite two layers approach, fondée sur une opposition esprit/ corps - d'une «natural, bodily, precultural emotion » et l'appréhender plutôt comme un « ideal, cognitive, cultural sentiment or second-order emotion ${ }^{17}$. Ce n'est pourtant pas le chemin suivi couramment par la sociologie française des religions, qui préfère voir dans l'émotion décrite par É. Durkheim l'expérience intense, ineffable, " extra-sociale ou au moins pré-sociale » du "contact émotionnel avec le principe divin » - la dimension sociale n'apparaissant que dans un second temps (ou "second palier »), quand "cette expérience se socialise et se rationalise, en se différenciant en croyances d'une part, et en cultes ou rites d'autre part. " ${ }^{18}$

16 Suivant cette approche, l'institution religieuse, en assurant la nécessaire « domestication » de cette expérience aussi intense qu'instable, l'inscrit dans des limites compatibles avec une vie sociale ordonnée, la convertit en une expression religieuse plus riche, plus élaborée mais aussi plus «froide». Elle s'expose ainsi à des mouvements de 
contestation revendiquant un retour à une religion "vraie ", débarrassée du primat moderne de la raison. Autrement dit, l'effervescence des émotions étant par nature la marque d'un état élémentaire et pré-social du religieux, toute religion émotionnelle peut certes légitimement prétendre renouer avec une sorte d'authenticité, mais elle entraîne inévitablement ses fidèles dans une régression, les ramenant à ce qu'était la religion avant sa socialisation, sa rationalisation, son institutionnalisation.

作 diversité et leurs contenus) hors du champ de l'analyse sociologique, pour se concentrer sur les conditions dans lesquelles s'opèrent la résurgence, l'endiguement ou l'institutionnalisation de l'émotion - envisagée comme un matériau «brut » et unique. Les réflexions d'É. Durkheim visaient pourtant le résultat inverse, en défendant l'idée que les émotions sont d'abord les produits d'un état social.

Faut-il alors considérer, comme y invite M. Nussbaum, que cette perspective théorique se rattache plutôt à une part de la tradition philosophique occidentale fondée sur deux lignes majeures d'objection aux émotions ${ }^{19}$ ? La première considère que « les émotions sont irrationnelles, au sens normatif du terme, car elles n'ont absolument rien à voir avec le jugement et la cognition. Ce sont des forces aveugles, provenant de quelque partie animale de notre nature qui ne participe pas au jugement. »Cette position absolue n'a que rarement été défendue par les grands philosophes occidentaux, qui ont notamment souligné le rôle de certaines émotions (« la peine, l'amour, la peur, la pitié », par exemple) dans la formation d'un jugement qui ne soit pas « aveugle ». Elle est, ajoute M. Nussbaum, «à présent largement discréditée même là où elle a été populaire - en psychologie cognitive par exemple et en anthropologie. Mais elle garde encore une emprise sur beaucoup de façons informelles de penser les émotions et d'en parler.»

La seconde ligne d'objection, nettement plus conséquente, réunit des philosophes pour qui les émotions ne «sont pas innées, mais socialement apprises, elles ne sont pas des pulsions aveugles, mais des opérations cognitives complexes. Cependant ces philosophes soutiennent que les jugements auxquels sont reliées les émotions sont tous faux. Ils sont faux parce qu'ils attribuent une très haute valeur aux personnes et aux événements extérieurs qui ne sont pas complètement contrôlés par la vertu et la volonté rationnelle de la personne. »C'est sur cette seconde ligne que se situent les observateurs du pentecôtisme associant expérience des charismes et émotion comme deux éléments d'un jugement faussé par des «évidences » trop aisément manipulables, auxquels ils opposent la stabilité qu'apporterait une compréhension plus « rationnelle » et mieux contrôlée de la Bible.

Il ne s'agit pas ici d'entrer dans une discussion de fond des conceptions philosophiques de l'émotion en Occident, mais plus simplement de souligner ce que la sociologie du pentecôtisme aurait à gagner en traitant cet héritage non comme un impensé implicitement investi dans l'analyse des religions émotionnelles, mais comme un point de vue particulier, européo-centré, consciemment assumé - ou rediscuté. Cette question a donné lieu, depuis deux décennies, à d'intenses débats au sein de l'anthropologie américaine ${ }^{20}$, qui s'est ainsi donné les moyens de repérer un certain nombre d'options théoriques tout à fait déterminantes pour l'étude des émotions, notamment en terrain pentecôtiste, telles que: matérialisme vs idéalisme, universalisme vs relativisme, romantisme vs rationalisme ${ }^{21}$. Ce faisant, elle a encouragé des tentatives de dépassement de ces stricts jeux d'oppositions, qui peinent trop souvent à cerner toute la complexité des expressions émotionnelles. Parmi ces tentatives, on mentionnera les réflexions de 
M.Z. Rosaldo, qui, en rapprochant connaissance et émotion, puis en reliant intensité émotionnelle et implication personnelle, fournissent à l'étude rapprochée des pratiques pentecôtistes des outils de grande portée heuristique :

(...) what distinguishes thought and affect - avançait-elle dans un article publié en 1984 -, differentiating a « cold » cognition from a « hot », is fundamentally a sense of the engagement of the actor's self. Emotions are thoughts somehow « felt » in flushes, pulses, « movements » of our livers, minds, hearts, stomachs, skin. They are embodied thoughts, thoughts seeped with the apprehension that «I am involved » ${ }^{22}$.

21 L'observation attentive du fonctionnement des Assemblées de Dieu polynésiennes incite précisément à ressaisir l'effervescence émotionnelle dans le cadre d'un dispositif institutionnel qui assure à la fois l'apprentissage de connaissances bibliques, de savoirfaire pratiques et leur mobilisation "chaude » au service d'une expérience religieuse vécue sur le registre de l'implication libre, forte et volontaire de chaque croyant dans sa relation personnelle avec Dieu. En restituant les rouages de ce travail institutionnel « invisible», on accède donc à un ensemble de "pensées incorporées » qui font apparaître les émotions exprimées par les fidèles non comme le débordement désordonné de sentiments "bruts», mais comme la mise en mouvement d'un ensemble de représentations préexistantes.

L'émotion des cultes pentecôtistes, où les croyants semblent s'abandonner à l'expérimentation «immédiate» du saint Esprit (et se soumettre du même coup à l'autorité de ceux qui leur paraissent les plus capables de susciter ces interventions surnaturelles) est vécue par eux comme une réponse spontanée à un ensemble d'évidences "vérifiées ». Ce point de vue "indigène », le sociologue ne serait pas loin de le partager, s'il supposait lui aussi que ces cultes ont en eux-mêmes une charge émotionnelle assez puissante pour produire, sans véritable préalable et par la seule force d'« évidences» (guérisons spectaculaires, échauffement des corps et des consciences, rythmes entraînants), des adhésions instantanées au pentecôtisme. Mais s'il est vrai que bon nombre des arguments déployés par l'évangélisation pentecôtiste ne reposent que sur une opération minimale de construction du sens et peuvent donc frapper les esprits les mieux disposés à croire, l'efficacité de ces démonstrations repose également sur des schèmes de compréhension plus complexes, diffusés notamment par le biais de l'évangélisation interpersonnelle. Plus encore, les sentiments exprimés par les fidèles réguliers des Assemblées de Dieu polynésiennes lors des différentes phases de la liturgie indiquent, de façon tout à fait explicite, la mobilisation de connaissances dispensées par un dispositif de formation efficace et cohérent, fondé sur une lecture spécifique de la Bible et orienté vers la mise en œuvre d'une relation «enchantée » entre le converti et Dieu.

23 La guérison miraculeuse, qui occupe une place primordiale dans le discours et les pratiques pentecôtistes, ne nécessite qu'une opération minimale d'interprétation, surtout lorsque l'évangélisation s'adresse à des individus dont l'attente croyante repose sur une compréhension culturelle de la maladie comme entité exogène. Il lui suffit de démontrer l'efficacité de ses rituels d'expulsion (prière, imposition des mains) pour convaincre qu'elle dit vrai. La guérison joue ainsi comme une évidence partagée, où chacun (même le plus étranger à l'univers pentecôtiste) est spontanément prêt à reconnaître l'intervention de puissances surnaturelles, la tâche de l'évangéliste consistant dès lors à relier cet 
événement à l'action d'un Dieu puissant et bienveillant qui ne demande rien en échange, si ce n'est que les miraculés lui « ouvrent la porte de leur cœur».

Les témoignages personnels, apportés par de simples convertis ou mobilisés par les pasteurs, constituent un second registre - au style plus indirect - de l'argumentation pentecôtiste, qui ne peut pleinement fonctionner qu'à deux conditions au moins : tout d'abord, un élargissement de la notion de "guérison », jusqu'à englober l'ensemble des symptômes du malheur ou du mal-être ; ensuite une propension à lire les détails de la vie quotidienne comme des signes d'influences surnaturelles (et la conviction que le Dieu chrétien participe directement à ces luttes d'influence). Le degré de familiarité avec ce type de croyances varie, bien entendu, selon les milieux socioculturels, mais l'adhésion " spontanée » à ces formes d'évidences dépend aussi du travail de socialisation réalisé en amont par les fidèles pentecôtistes auprès de leurs amis, de leur famille ou de leurs collègues de travail. En effet, l'invitation d'un proche - qui semble (toutes les études s'accordent sur ce point) une voie privilégiée d'accès au pentecôtisme - est acceptée d'autant plus volontiers que celui-ci aura su auparavant démontré comment Dieu a transformé sa vie et convaincre que « ce qu'il a fait pour moi, il peut le faire pour toi ». Au sein des Assemblées de Dieu polynésiennes, cette capacité d'argumentation et d'identification est inculquée méthodiquement dans le cadre d'un programme de formation à la "vie chrétienne ", suivi par la plupart des convertis.

Enfin, un troisième registre, autour du thème de la "chaleur", mérite un examen particulier car il est celui où les conceptions « indigènes » et les « notions anticipées » sur l'émotion pentecôtiste se rejoignent le plus complètement. Dans leurs récits de conversion, les fidèles décrivent, avec une grande régularité, une «chaleur » qui, tel un souffle parcourant l'assemblée, les a envahis, leur a tiré des larmes irrépressibles et les a finalement convaincus de répondre, en quelque sorte malgré eux, à l'appel. Une femme dit par exemple: "Au moment où il [l'évangéliste] appelle les gens à se lever pour l'imposition des mains, je veux pas me lever. Tout d'un coup, une chaleur venait de mon pied, m'a poussée, je me suis levée. ${ }^{23}$ Une autre évoque un effet d'entraînement collectif: "J'ai senti, mon cœur c'est comme ça, ça va éclater, je dois aller devant ou rester. J'ai ouvert les yeux, mon beau-frère est parti [devant]. Je suis partie devant avec les autres, je suis allée devant, je suis restée comme ça, c'était la première fois que je vois cette religion (...). On dirait je flotte, je suis obligée de partir devant. » ${ }^{24}$

Une lecture de l'émotion pentecôtiste comme "émotion des profondeurs » pré-sociale retrouverait sans mal dans ces descriptions tous les traits de l'enthousiasme élémentaire que suscite le rassemblement communautaire, accentué par le rythme entraînant des chants, la vision de miraculés, la contagion émotionnelle entretenue par l'évangéliste et les témoignages personnels. Et indéniablement, les réunions d'évangélisation produisent, presque mécaniquement, un certain nombre de stimulations agissant notamment sur le rythme cardiaque, le système nerveux ou activant des réflexes compassionnels. Mais il faut aussi, pour saisir réellement ce qui, dans cette "chaleur ", conduit à l'adhésion religieuse, revenir à la dimension d'implication personnelle évoquée par M. Z. Rosaldo : quelles sont les connaissances et les espérances préalables qui, mobilisées ainsi «à chaud", conduisent des individus à la certitude d'avoir découvert la voie du salut? Les données recueillies au sein des Assemblées de Dieu polynésiennes apportent quelques clés de compréhension pour échapper à la fois à une compréhension "enchantée » et au paradigme de la régression pré-sociale. 
27 En premier lieu, le comportement (souvent inaperçu) des membres réguliers des assemblées qui assistent en masse aux réunions d'évangélisation (ils constituent entre un tiers et un quart $d u$ "public »), joue un rôle déterminant, parce qu'il est interprété comme le signe d'une différence profonde qui ne peut être feinte. Chaque converti s'efforçant de devenir un témoignage vivant (et transparent) du salut, ce comportement contribue fortement à convaincre les nouveaux venus qu'ils se trouvent dans « un lieu saint ", tandis que l'argumentation pentecôtiste leur rappelle combien eux-mêmes sont marqués par le péché. Et c'est dans cet écart entre la découverte d'une "sainteté » aussi remarquable qu'enviable et sa propre indignité, entre la volonté d'en être et le sentiment que c'est impossible, que se noue l'implication du croyant dans une démarche personnelle d'adhésion. On le voit bien à travers le récit qu'en fait Daniel, l'émotion (la " chaleur») précédant l'adhésion renvoie à une construction de sens qui ne peut être réduite à un simple effet d'exaltation collective :

J'ai commencé à sentir une chaleur - se souvient-il -, une présence, à me sentir bien et en même temps je me sentais sale. J'avais l'impression d'être dans un lieu saint, que tout le monde était saint, j'entendais une voix intérieure qui disait « je suis là, je t'aime, je t'accepte tel que tu es ». Et j'ai essayé de me retenir de pleurer, mais je ne pouvais pas, je pleurais de joie et je pleurais de repentance. Je croyais que je n'étais pas digne de m'approcher de Dieu, je m'étais trompé et là, je me sentais accepté (...). Et je ne voyais personne autour de moi, j'avais l'impression d'être dans un autre lieu, d'être au milieu des anges. Cette rencontre personnelle avec Dieu, ça continue toujours, mais c'était la plus belle, cette fois-là ${ }^{25}$.

Bien plus qu'une simple mise en intrigue attribuant a posteriori un sens à ce qui n'était qu'une expérience émotionnelle « élémentaire », ce récit permet d'entrevoir un ensemble assez cohérent de représentations spécifiquement pentecôtistes, qui apparaissent ici aux prémisses de l'adhésion et sont mobilisées, sous une forme plus nette et mieux consolidée, par les participants réguliers aux cultes dominicaux des Assemblées de Dieu polynésiennes. Comme l'indique déjà le récit de Daniel, cette expérience émotionnelle se construit à l'articulation entre l'enthousiasme militant de la communauté croyante et l'engagement de chacun dans une relation très personnelle avec Dieu.

En entrant dans l'église alors que le culte commence, on est frappé par l'enthousiasme avec lequel les fidèles entonnent les chants, tapant des mains, levant les bras ou imitant les mouvements d'une marche. On remarque le plaisir visible que chacun prend à retrouver ses "frères et sœurs en Christ » : sourires, embrassades et gestes d'affection que l'on s'échange encore tandis que les officiants ont déjà lancé les premiers chants. Et en effet, la première séquence du culte est celle du "nous ", animée par des chants célébrant la joie d'une réunion fraternelle («nous voici réunis, comme une famille/et nos mains sont unies »; « quelle joie, quelle joie, Seigneur, d'être là aujourd'hui ») et la force d'une assemblée combattante, en marche vers la victoire :

Assaillons les villes, franchissons les murailles

Nous sommes l'armée de Dieu qui combat dans les cieux.

Cet enthousiasme visible est interprété par les fidèles réguliers comme une première manifestation du saint Esprit - celui-ci étant précisément la forme symbolique sous laquelle ils se représentent l'action salvatrice que Dieu (par le biais de dispositifs « invisibles » de socialisation, de formation et de contrôle communautaire) exerce dans leur existence personnelle. Autrement dit, cette puissance du saint Esprit, qui est «avec eux » (ce que confirmeront, dans la suite du culte, les guérisons, parlers en langues ou dans des églises plus charismatiques - transes, " rire dans l'esprit », etc.) est aussi " en 
eux ", comme voix intériorisée d'un ethos pentecôtiste. L'enthousiasme déclenché par cette première séquence du culte est donc celui d'une communauté qui se retrouve et se reconnait comme telle, en même temps que celui d'individus croyants persuadés d'entrer en communication directe avec la « puissance bienveillante » du saint Esprit qui, habitant les convertis, chaque jour les assiste, les protège et leur assure un sort privilégié ${ }^{26}$. Et lorsque « l'armée de Dieu » se tourne, au terme de cette séquence, vers le prédicateur afin qu'il lui indique vers où marcher et qui combattre, c'est vers cette dimension individuelle de l'expérience pentecôtiste qu'insensiblement, il oriente les fidèles : «où en es-tu, ce matin, de ta relation personnelle avec Dieu? » Cette deuxième séquence qui s'ouvre avec la prédication est ainsi celle du «je», marquée par des chants de louange aux rythmes plus lents, tous conjugués à la première personne du singulier. Elle est aussi, et de loin, celle où l'émotion s'exprime avec le plus d'intensité et dans la plus grande effervescence : dans ce dialogue intime avec Dieu, chacun veut dire ce qu'il a «sur le cœur » et toutes ces paroles mêlées forment un brouhaha à peine ponctué par les «amen! » et «alléluia!» que lancent de temps à autre les officiants. Une double certitude est au principe de cette volonté éperdue de communiquer personnellement avec Dieu : l'assurance qu'il est entré dans la vie de chacun - «tu as touché mon cœur, tu m'as sauvée, tu es tout ce que j'ai » dit par exemple une fidèle de Tahiti - et la conviction que la ferveur atteinte lors des cultes lui permet d'être présent plus que jamais, de telle sorte que le croyant peut espérer y recevoir les messages que Dieu lui destine.

31 L'intensité de ces expressions émotionnelles repose donc sur la mobilisation "chaude " de deux ensembles de dispositions spécifiques: d'une part, une compréhension du message biblique focalisée sur l'action bienveillante d'un Dieu personnel et l'implication de chaque fidèle dans une relation intime dont l'authenticité tient à l'absence (apparente) de toute médiation (institutionnelle ou communautaire) ; d'autre part une capacité à lire les événements et les discours produits lors du rassemblement communautaire sur un mode très personnel, comme autant de messages adressés par un Dieu qui «connaît le cœur de chacun ». On le voit, au-delà de l'effervescence apparemment désordonnée des cultes, les réactions des fidèles réguliers des Assemblées de Dieu polynésiennes traduisent moins une régression de la religion vers ses formes les plus élémentaires qu'une articulation complexe entre personnalisation des croyances, Dieu proche et encadrement institutionnel - articulation qu'il convient d'interroger en remontant à sa source, c'est-àdire aux dispositifs de socialisation et de formation. Car les proclamations de la toutepuissance du saint Esprit, invitant les fidèles à «se laisser former par Dieu » s'accompagnent - au sein du pentecôtisme «classique» au moins - d'actions de formation systématiques, le plus souvent orientées vers des prescriptions éthiques et des savoir-faire plutôt que vers des formes de savoir académiques.

Ces programmes de formation à la "vie chrétienne", qu'ils soient d'origine institutionnelle (comme les programmes de l'International Correspondance Institute des Assemblées de Dieu américaines) ou non dénominationnelle (comme la méthode INSTE ${ }^{27}$ utilisée par les Assemblées de Dieu polynésiennes), s'efforcent de concilier, par leur méthode et leur contenu, la soumission du fidèle au saint Esprit et l'effort de formation individuelle. La méthode, fondée sur un modèle horizontal d'enseignement mutuel et de «partage des expériences» plutôt que sur un dispositif d'instruction vertical et dissymétrique, substitue à la figure du maître celle du mentor, animateur laïque dont la légitimité institutionnelle (il est désigné par les pasteurs) s'efface au profit de l'autorité amicale que lui vaut un parcours personnel exemplaire (exemplarité qui, elle-même, 
provient, pour une large part, de la reconnaissance "invisible», que lui confère l'institution à travers le rôle de mentor).

Le contenu vise à faire entrer le converti dans une relation « enchantée » avec Dieu, en lui apprenant d'une part "à se connaître » - c'est-à-dire à distinguer sa " nouvelle identité » (ce qu'il est devenu par la conversion) de sa "vieille nature » (ce qu'il est encore malgré la conversion) - et d'autre part à « connaître Dieu » - en sachant entendre sa voix, à travers les circonstances, les conseils et la lecture assidue de la Bible. Par cet effort de connaissance, les fidèles acquièrent une réelle pratique de la parole biblique (comprise dans un sens littéral et pragmatique) ainsi qu'un savoir-faire communicationnel (écouter, se confier, argumenter) et se donnent les moyens d'une transformation profonde de leur existence en s'assignant un devoir personnel de témoignage.

De telles formes de socialisation et de formation marquent, à leur manière, le déplacement contemporain « du lieu de la vérité du croire, de l'institution vers le sujet croyant ${ }^{28}$ : si l'institution intervient objectivement (en préférant l'enseignement à la discipline, le conseil amical et le " partage » au rappel à l'ordre) dans la reconfiguration des vies personnelles, elle le fait par un travail « invisible » amenant les convertis à vivre une expérience subjective entièrement centrée non sur l'insertion obligée dans une église, mais sur la relation à Dieu. Ainsi, l'intériorisation d'un devoir de témoignage et d'évangélisation est vécue comme une réponse délibérée du croyant à la volonté de Dieu, qui se fait entendre à travers les Écritures (lues comme autant de messages que l'on prend pour soi) et se révèle par les « dons » particuliers qu'il accorde à chacun (révélation dans laquelle l'institution intervient comme instance «invisible» de discernement et de certification). Les conseils prodigués par les pasteurs, les discours normatifs diffusés par les prédications et le contrôle des "frères et sœurs en Christ " ne sont jamais reçus autrement - lorsque l'«enchantement» fonctionne pleinement - que comme des messages « de la part de Dieu ».

Ces programmes de formation, expression systématisée et assez explicite d'un corpus de doctrines et de pratiques spécifiques, permettent d'identifier avec précision le point à partir duquel effervescence émotionnelle et rationalisation des vies personnelles peuvent être ressaisies comme deux dimensions d'un même paradigme religieux, que nous proposons d'appeler «évangile relationnel ${ }^{29}$. Dans son analyse du terrain réunionnais, B. Boutter l'a très justement souligné, l'adhésion au pentecôtisme fait entrer le croyant dans un «système d'interactions complémentaires entre l'individu et Dieu » qui soumet l'ensemble de ses relations - y compris les conflits psychologiques de l'individu avec luimême - à la médiation de Dieu ${ }^{30}$. L'instauration de cette communication constante et transparente engendre une reconfiguration profonde des identités personnelles, des modes de vie et de l'expérience religieuse.

Dans le rapport que l'individu entretient avec ses propres conflits tout d'abord, l'intériorisation de normes et de connaissances, sous la forme d'une "voix du saint Esprit ", qui devient " plus que la conscience dans une vie ${ }^{31}$ en produisant des rappels à l'ordre sans intervention visible de l'autorité institutionnelle, autorise une distinction entre la "vieille nature » et la "véritable identité ». La conversion, entendue comme le moment d'une re-naissance "en Christ», assigne en effet au converti un devoir d'exemplarité, qui lui enjoint d'une certaine manière d'être d'emblée ce qu'il veut devenir, afin de porter témoignage de ce que « Dieu peut faire dans une vie ». Pris dans cette tension entre le devoir-être et l'être, l'individu s'en remet à la médiation de Dieu : ces pensées, ces intentions qui lui viennent sont-elles « de Lui »? sont-elles au contraire 
imputables à cette autre part de lui-même qu'il entend désormais tenir à distance ou à l'esprit du " monde » ? Pour les croyants engagés dans ce dialogue avec eux-mêmes et aux prises avec la complexité quotidienne de l'existence, la communication est la solution : il faut « rester en ligne».

Au cours de la journée - expliquent les auteurs du programme INSTE -, nous nous retrouvons souvent avec des personnes ou dans des circonstances qui nous contrarient. Dans ce cas, nous devons mettre en pratique le «Priez sans cesse » avec les prières «SOS ». Et si nous sommes «restés en ligne " avec Dieu, nous pouvons prier « Seigneur, aide-moi à avoir ton attitude envers cette personne ou cette circonstance ", et à ce moment, le Seigneur nous répondra ${ }^{32}$.

Les interpellations $d u$ «monde» et les réactions spontanées se trouvent donc systématiquement soumises à la médiation de cette relation constante et transparente entre le croyant et Dieu. Concrètement, les communications «de la part de Dieu » empruntent différents canaux qui traduisent tous l'insertion du converti dans une communauté croyante et dans un dispositif institutionnel resserré. Ainsi, un fidèle de Tahiti, avant de se lancer dans la culture de la vanille, s'assure qu'il s'agit bien là de la volonté de Dieu :

Et il m'a répondu, à travers des messages, la Bible, des prophéties, l'église plusieurs fois, des gens qui disent « fais ci, fais ça »-, le pasteur et les circonstances [c'est-à-dire des faits précis, mais surtout, les interprétations légitimes de ces faits qu'apportent la communauté et les pasteurs]. J'ai eu ces quatre réponses: la Bible, l'église, le pasteur et les circonstances. Et donc j'ai su que c'était ça, maintenant je sais que je vais faire ça ${ }^{33}$.

Ce dispositif communicationnel contribue à une modération des comportements, plus réfléchis, moins réactifs car moins directement en prise avec les enjeux mondains. Il a trois implications majeures: il passe par l'instauration, au sein de la communauté croyante, d'un mode de relation interpersonnelle "enchanté»; il s'appuie sur l'intervention de pasteurs détenteurs d'un savoir-communiquer et agissant en «montreurs de communication»; enfin, il transforme le culte en un espace de communication de haute intensité, où des manifestations exceptionnelles de la présence divine répondent à une expression de soi totale, libérée de toute restriction ou formalité.

«Bien que ta décision de suivre Christ ait été personnelle, la vie en Christ est très relationnelle », précise le programme INSTE, car la communion au sein de la «famille de Dieu » est un «moyen de croissance» spirituelle ${ }^{34}$. La médiation «invisible» d'une communauté croyante fondée sur un idéal de communication transparente (qui incite à « dire vrai » et à tout se dire) et sur un devoir de témoignage (qui assigne à chacun une identité «vraie») apporte en effet aux convertis - selon l'expression d'un pasteur français, "le contenu du salut dans l'humanité» ${ }^{35}$, qui comprend trois éléments principaux. En premier lieu, la communauté joue, au côté de l'encadrement pastoral, un rôle essentiel dans la normalisation des comportements individuels : en autorisant la confidence, elle permet à chacun de faire connaître à Dieu ce qu'il a «sur le cœur » et assure en retour la diffusion des réponses divines, c'est-à-dire de normes pentecôtistes reçues par les fidèles sur le mode d'une communication intime nouée avec un Dieu qui " connait le cœur de chacun » et " répond toujours aux prières ». Les convertis s'efforcent d'instaurer entre eux une " communication du quatrième niveau ", en acceptant de "se révéler l'un à l'autre, c'est-à-dire révéler quelque chose qui ne pourrait se savoir si on ne le disait pas ; par exemple, un combat spirituel, une perception personnelle, un projet ou une crainte. ${ }^{36}$ Ces confidences deviennent, pour les membres de la communauté 
croyante, autant de «fardeaux » que Dieu «met sur leur cœur » et qu'ils incluent dans leurs prières, à travers une démarche d'implication personnelle qui est aussi un signe de soutien indirectement adressé aux intéressés, selon un principe bien décrit par Éliane :

C'était dur avec mon mari, lui allait à ses dîners, ses soirées politiques, il ne s'inquiétait pas de moi. Mais je sais que les frères et les sœurs priaient beaucoup pour moi, pour que je vienne au culte en paix. J'entendais leurs prières, mais jamais ils ne venaient me voir pour me dire " ton mari, ci, ça ». Il y a beaucoup de réunions de prière, $j$ 'arrivais et $j$ 'entendais des prières pour moi ${ }^{37}$.

Ensuite, l'harmonisation produite par le devoir de témoignage constitue la communauté en un « îlot d'humanité » ${ }^{38}$ ) où la prévisibilité des réactions et la redondance des signes d'apaisement, contrastant avec la relative insécurité des relations sociales ordinaires, confirment la présence d'un Dieu protecteur et accueillant : « tu te gênes pas quand tu viens ", résume une fidèle polynésienne ${ }^{39}$. Enfin, ce modèle relationnel, que la communauté pentecôtiste s'efforce de mettre en œuvre, apporte aux fidèles une référence pratique et des compétences qu'ils investissent spontanément dans leur vie familiale, sociale et professionnelle. L'adhésion au pentecôtisme entraîne, assez inévitablement, des tensions avec l'entourage familial non converti et une rupture des relations sociales trop ancrées dans "le monde ". Elle fournit aussi aux convertis des ressources symboliques leur permettant de renouer ou dénouer des liens anciens, en surmontant les obstacles dressés jusque-là par la logique culturelle d'honneur - très prégnante en Polynésie française ${ }^{40}$. Anita explique comment elle a pu se libérer de «l'emprise de son adversaire»:

Il y avait une personne dans ma vie que je n'arrivais pas à aimer, quelqu'un qu'il n'était pas question de voir, j'avais de la haine pour cette personne. J'ai appelé cette personne, on a eu un entretien, je lui ai demandé pardon, tout ça, je pouvais pardonner, alors que je ne pouvais pas, avant. (...) c'était ça, mon problème, j'avais ce poids, de la haine, j'étais sous l'emprise de mon adversaire, je ne pouvais pas pardonner ${ }^{41}$.

41 Selon les termes de cet «évangile relationnel », ce n'est pas l'individu lui-même qui, en «s'abaissant» ainsi, perd la face, mais Dieu qui agit en rétablissant un dialogue, une communication transparente d'où le converti sort vainqueur. Nombre de fidèles, qui tentent à l'inverse (au nom d'un idéal pentecôtiste d'harmonie familiale) de renouer des liens avec des parents auxquels les opposaient des différends insurmontables, s'appuient sur le même principe pour « repartir à zéro ".

Les formes de l'autorité s'en trouvent sensiblement modifiées, entre époux comme entre parents et enfants, car un conflit ne doit plus se résoudre en « [usant] d'autorité pour imposer une solution ", mais par une discussion ouverte (dans laquelle Dieu intervient comme médiateur) qui, seule, permettra de rétablir la relation «brisée» (car tout problème est une "relation brisée ${ }^{42}$ : «Il faut dialoguer - dit un pasteur - et en dialoguant, on évite les problèmes ${ }^{43}$ ). Ce qui ne signifie pas nécessairement l'établissement de relations égalitaires - en particulier entre parents et enfants - mais plutôt un glissement vers une autorité "amicale" recherchant par le dialogue un consentement volontaire à la domination. En outre, cette "bonne volonté communicationnelle» dote les convertis de ressources symboliques spécialement en affinité avec l'idéologie moderne de la «société de communication » et donc susceptibles de «porter des fruits» dans leur vie sociale et professionnelle (tout spécialement dans l'économie des services aux personnes). La capacité de "bien parler ", de "se dire » et l'aptitude à traduire des situations de conflit en termes de "problèmes de 
communication", qui sont inculquées à des croyants soucieux d'établir une relation authentique avec Dieu et de participer efficacement à la "grande mission", produisent ainsi indirectement les preuves expérimentales d'une protection divine s'étendant à tous les domaines de l'existence.

Le pasteur s'inscrit au cœur de cet évangile relationnel en tirant sa légitimité spécifique d'une compétence - personnelle et professionnelle - de «montreur de communication » ${ }^{44}$, qui se déploie sur les deux versants de l'expérience pentecôtiste : la mise en ordre des vies personnelles, par un travail de suivi personnalisé, et la mise en forme des cultes comme moments de haute intensité communicationnelle. Parce qu'il apparaît aux croyants comme un spécialiste en communication (avec Dieu et, par médiation, avec soi et autrui), il est celui qui peut les aider à traduire leurs problèmes en termes de « déficit d'information, d'échange, d'écoute " ${ }^{45}$. Il identifie, par la prière à haute voix et la confidence, les obstacles, les non-dits, les influences parasites et restaure ainsi la transparence, la fluidité des échanges avec Dieu, «s'effaçant» finalement, lorsque la relation intime entre le croyant et son Dieu est pleinement rétablie.

Acteur central du spectacle cultuel, il y met en scène un dialogue direct, vivant - avec l'assemblée comme avec Dieu - à travers une prédication reçue comme un discours à la fois spontané ("sans façon», sans trace visible de fabrication) et révélé (un message de Dieu pour l'assistance) :

A good Pentecostal pastor - écrit W. J. Hollenweger - does not preach a sermon. The written text of theological or exegetical preparation does not come between him and his congregation. He is not a «man of fine phrases » (...) but he is never at loss for words (...). He allows the social background of his hearers to «put him off » and in fact these play a great part in determining the content and form of what he says. «A good Pentecostal preacher is well worth hearing, for he has a genius for communication; his preaching is not a lecture but a dialogue $»^{46}$.

Le pasteur est aussi, sur un registre moins explicite, celui qui facilite et encadre la libre communication entre les fidèles et Dieu, dans cet espace où plus rien - règles de la bienséance sociale, usages normés du langage et codes de tenue corporelle - n'est censé faire obstacle à une rencontre immédiate, intense. Il intervient ainsi comme seule instance légitime de discernement des manifestations surnaturelles, authentifiant les signes et les messages qui sont "de l'Esprit ", disqualifiant ce qui lui paraît au contraire relever du seul dérèglement nerveux ou d'influences maléfiques. Surtout, il est le garant institutionnel d'une libération de la parole individuelle qui repose sur un décentrement radical de la référence normative. En effet, la conformité des paroles prononcées ne se mesure plus par rapport à une norme transcendante (la prononciation conforme des "mots de Dieu», paroles consacrées exprimant le respect de son autorité), mais par rapport à l'individu lui-même : a-t-il dit tout ce qu'il avait «sur le cœur »? Dans une société polynésienne historiquement marquée par une culture de l'oralité, où la prise de parole est encore aujourd'hui un enjeu social, politique et religieux de premier ordre, ce renversement produit des effets non négligeables, que les convertis vivent individuellement comme une victoire contre la " timidité ». Mais cette timidité est moins psychologique que sociale, et traduit plus précisément la force de plusieurs mécanismes d'intimidation : en particulier, la sacralisation de la langue tahitienne ( $r e^{\prime} \mathrm{o} \mathrm{ma} \mathbf{a}^{\prime} \mathrm{hi}$ ) comme langue biblique (dont l'authenticité est protégée par la quasi-immuabilité de la traduction tahitienne de la Bible établie en 1838 par les missionnaires anglais ${ }^{47}$ ) par le protestantisme traditionnel, combinée à la concurrence du système éducatif français, décourage les plus jeunes de s'exprimer dans une langue tahitienne qu'ils maîtrisent 
assez mal (évitant par là de commettre une double transgression, religieuse et culturelle), tandis que les plus âgés renoncent facilement à prendre la parole en français (convaincus qu'ils le «parlent mal»). Si le pentecôtisme introduit là une rupture spectaculaire, il le fait au prix d'un évidement de la parole qu'il libère: c'est d'abord l'expression qui importe et l'on suit la volonté de Dieu dès lors que l'on dit les choses «comme elles viennent ». Le pasteur s'efforce donc, par une sorte de maïeutique (« sentez vous libres de louer le Seigneur ", "que chacun élève sa voix vers Dieu ", etc.), de désamorcer les inhibitions qui empêchent chacun de « se dire».

Si dans vos prières, vous ne savez pas quoi dire, ceux qui sont baptisés du saint Esprit, parlez en langues, et le saint Esprit parlera pour vous. (...) si vous êtes dans l'adoration, on ne sait pas quels mots dire, parlez en langues, le saint Esprit va faire monter vers Dieu des paroles ineffables ${ }^{48}$.

On le voit, l'établissement d'une communication « vérifiée » avec Dieu prime alors, in fine, sur le contenu même de ce qui est échangé, selon un processus défini par l'école de Palo Alto comme une « métacommunication »:

Quand A communique avec B, le simple fait de communiquer peut comporter l'énoncé implicite : «nous sommes en train de communiquer ». En fait, cet énoncé peut être le message le plus important qui soit émis et reçu ${ }^{49}$.

Comme l'a bien noté W.J. Hollenweger, les cultes pentecôtistes atteignent ainsi un « degré incroyable de communication »:

In Pentecostal worship - which only a casual observer could describe as unstructured and unliturgical - everyone can express himself with the means of speech at his own disposal. The criterion is not conceptual clarity, but communicability ${ }^{50}$.

C'est ici sans doute que se joue une bonne part du «risque pentecôtiste », si l'on entend par là un basculement possible de l'expérience religieuse vers une expérimentation charismatique se suffisant à elle-même, où le débordement de l'expression évacue toute construction du sens, toute argumentation théologique. « Reste que - comme le remarque très justement J.-P. Willaime - s'il n'y a pas de dimension éthique, on est alors dans un autre type de religion. Le pentecôtisme, en tant qu'il constitue une des expressions du christianisme, appartient en effet au monde des religions éthiques. ${ }^{51} \mathrm{Et}$ cela ne se voit nulle part plus nettement qu'à travers une pratique aussi exemplaire que la glossolalie, souvent mobilisée pour démontrer le caractère inarticulé et «émotionnel » du discours pentecôtiste, mais qui, si on l'analyse attentivement, met à jour, tout au contraire, l'articulation entre discours éthique, travail institutionnel et expressions émotionnelles. En effet, lorsqu'elle n'est pas une simple " prière en langues » (c'est-à-dire, on l'a vu, une modalité d'expression totale de soi à Dieu, libérée des contraintes ordinaires du langage) mais est reçue par l'assemblée comme une communication de Dieu, elle met en branle un ensemble de mécanismes "invisibles" ayant peu à voir avec une improvisation désordonnée.

Elle est d'abord un énoncé relativement bref et maîtrisé, prononcé d'une voix forte par un fidèle conscient d'avoir, à travers l'exercice légitime de ce don personnel (certifié par l'encadrement pastoral et validé par la communauté croyante), un rôle à jouer dans l'église. Son authenticité n'est reconnue que si la glossolalie est suivie d'une interprétation, par laquelle un second fidèle (toujours sur la base d'un don personnel et légitime) délivre le message adressé par Dieu à des personnes qui se reconnaîtront (ou que l'on reconnaîtra). Il s'agit essentiellement de paroles de réconfort et de rappels à l'ordre : 
(...) Tu as endurci ton cœur, tu sais que l'orgueil précède la chute. Je t'appelle encore ce matin, repens-toi, reviens vers moi, pour ta vie spirituelle, pour ta vie de foyer, mon cœur est triste de te voir, tant de fois, tant de fois je t'appelle, viens parce que je $t^{\prime}$ aime ${ }^{52}$.

Nourries d'une familiarité spécifique avec le texte biblique - paroles et conseils divins que l'on "prend pour soi ", comme autant de réponses à des difficultés ou des hésitations ces interprétations sont comprises par les fidèles comme une vérification expérimentale d'une « onction divine » de l'assemblée et de la relation personnelle établie entre chaque converti, "resté en ligne», et Dieu. Car la médiation, assurée par l'interprète disparaissant derrière la perception "enchantée » d'une communication directe avec Dieu (spectaculairement attestée par l'irruption d'un langage surnaturel), les fidèles ont alors la certitude d'entendre Dieu. La glossolalie et son interprétation concentrent ainsi les éléments clés de la théologie pentecôtiste: une normalisation des vies qui passe par l'instauration d'une relation « enchantée » avec Dieu, où les pasteurs et les « frères et sœurs en Christ» interviennent comme médiateurs «invisibles»; des temps de communication intense et émotionnelle avec Dieu, au cours desquels il manifeste la constance et la force de son engagement au côté du converti.

51 Au-delà du paradoxe apparent que constitue la coexistence, au sein d'un même espace religieux, d'une forte effervescence émotionnelle et d'une rationalisation des vies personnelles, c'est donc bien à partir d'un paradigme religieux cohérent - un " évangile relationnel», en forte affinité avec les idéaux modernes de la «société de communication" - plutôt qu'en postulant simplement son caractère régressif et élémentaire, que l'on peut véritablement rendre compte de la complexité de l'expérience vécue par les fidèles des Assemblées de Dieu.

\section{NOTES}

1. Harvey Cox, Retour de Dieu. Voyage en pays pentecôtiste, Paris, Desclée de Brouwer, 1995, p. 26.

2. Jean-Paul Willaime, « Le pentecôtisme : contours et paradoxes d'un protestantisme émotionnel », Archives de Sciences Sociales des Religions, $\mathrm{n}^{\circ}$ 105, 1999, p. 13-14.

3. Sébastien Fath, «L'autorité charismatique au cœur de l'Église : pentecôtisme et débat sectaire ", Études théologiques et religieuses, $\mathrm{n}^{\circ}$ 3, 2001, p. 371-390.

4. Martha Nussbaum, «Les émotions comme jugements de valeur ", in Patricia Paperman et Ruwen Ogien, La couleur des pensées. Sentiments, émotions, intentions, Paris, éditions de l'EHESS, 1995, p. 19-32.

5. Les résultats de cette enquête ont constitué le principal matériau d'une thèse de doctorat en sociologie soutenue en février 2004 à l'EHESS et publiée en 2005 : Yannick Fer, Pentecôtisme en Polynésie française, l'Évangile relationnel, Genève, Labor et Fides, 2005.

6. Émile Durkheim, Les règles de la méthode sociologique, Paris, PUF, 1987, p. 142.

7. Cf. notamment Alain Babadzan, Naissance d'une tradition. Changement culturel et syncrétisme religieux aux îles Australes (Polynésie française), Paris, ORSTOM, 1982 ; Yannick 
Fer, Gwendoline Malogne-Fer, Tuaro'i, réflexions bibliques à Rapa. Conversion et identité, Papeete, Haere po, 2000.

8. Jean-Pierre Bastian, « De l'autorité prophétique chez les dirigeants pentecôtistes », Revue d'histoire et de philosophie religieuse, $\mathrm{n}^{\circ}$ 2, 2001, p. 200.

9. Gilles Rivière, « Le pentecôtisme des hauts-plateaux boliviens ", Problèmes d'Amérique latine, $\mathrm{n}^{\circ}$ 24, 1997, p. 91.

10. Danièle Hervieu-Léger, « Renouveaux émotionnels contemporains. Fin de la sécularisation ou fin de la religion? » in Françoise Champion, Danièle Hervieu-Léger, dirs., De l'émotion en religion. Renouveaux et traditions, Paris, Centurion, 1990, p. 222. 11. Émile Durkheim, Les formes élémentaires de la vie religieuse. Le système totémique en Australie, Paris, PUF, 1994, p. 603.

12. Ibid., p. 312.

13. Ibid., p. 308.

14. Ibid., p. 327.

15. Ibid., p. 308.

16. Ibid., p. 315.

17. Catherine LUTZ, Goeffrey WHITE, «The Anthropology of Emotions ", Annual Review of Anthropology, 15, 1986, p. 407. Nous adressons ici nos remerciements à Patrice Mann pour nous avoir indiqué cette précieuse référence.

18. Danièle Hervieu-Léger, 1990, op. cit., p. 220-221.

19. Martha NusSBAuM, 1995, op. cit.

20. Catherine LuTZ, Goeffrey WhiTE, op. cit.

21. Cette dernière opposition théorique constitue, par exemple, le fil conducteur retenu par André Corten, analysant le pentecôtisme brésilien sous l'angle d'un « romantisme théologique ", qui amène ses fidèles à la piété en privilégiant la ferveur émotionnelle et les sentiments contre la raison et les élaborations doctrinales (André Corten, Le pentecôtisme au Brésil. Émotion du pauvre et romantisme théologique, Paris, Karthala, 1995). 22. Michele Z. ROSALDO « Toward an anthropology of self and feeling ", in R.A. SHWEDER, R.A. LE VINE, dirs., Culture Theory: Essays on Mind, Self and Emotion, Cambridge, Cambridge University Press, 1984, p. 143.

23. Assemblée de Dieu de Tahaa (îles Sous-le-Vent), entretien du 4 juin 2001.

24. Assemblée de Dieu de Faa'a (île de Tahiti), entretien du 13 décembre 2000.

25. Assemblée de Dieu de Faa'a, entretien du 7 novembre 2000.

26. À propos de la société, É. Durkheim écrit que l'homme « ne pouvait donc échapper à ce sentiment qu'il existe en dehors de lui des causes agissantes d'où lui viennent les attributs caractéristiques de sa nature, et comme des puissances bienveillantes qui l'assistent, qui le protègent et qui lui assurent un sort privilégié. Et à ces puissances il devait nécessairement attribuer une dignité qui fût en rapport avec la haute valeur des biens qu'il leur attribuait. » (1994, op. cit., p. 303).

27. «Institut de théologie par extension », programme développé à partir de 1982 par l'association Open Bible Standard Churches. Les Assemblées de Dieu polynésiennes utilisent le premier niveau, intitulé " préparation au service », qui comprend quatre livres et débute par Formation du disciple. En suivant Jésus dans un service fidèle (Nicolas et Lena Venditti, Open Bible Standard Churches/Department of International Ministries, Iowa, 1996).

28. Danièle Hervieu-Léger, La religion pour mémoire, Paris, Cerf, 1993, p. 247.

29. Yannick FER, 2005, op. cit., p. 368 ss. 
30. Bernard Boutter, La mission Salut et Guérison à l'île de la Réunion. Contribution à une anthropologie du pentecôtisme dans les sociétés en mutation, thèse de doctorat en ethnologie, Université Strasbourg II, 1999, p. 251 ss.

31. «Il est là, explique Marthe, et quand vous allez faire quelque chose qui n'est pas conforme à la parole de Dieu, il va vous montrer, vous prévenir que non, c'est pas comme ça. C'est plus que la conscience, le saint Esprit dans une vie. » (Papeete, entretien du 2 avril 2001).

32. Nicolas et Lena Venditti, op. cit., p. 127.

33. Assemblée de Dieu de Faa'a, entretien du 7 novembre 2001.

34. Ibid., p. 237.

35. Pasteur Jean-Pierre Charlet, entretien du 10 février 2003.

36. Nicolas et Lena Venditti, op. cit., p. 284-285.

37. Assemblée de Dieu de Papeete, entretien du 7 mai 2001.

38. An « Island of Humanity » (Walter J. Hollenweger, The Pentecostals, Peabody (Mass.), Hendrickson Publishers, 1988, p. 457 ss).

39. «Ce que j'aime bien, dans l'Église pentecôtiste, tu voyais les gens, leurs visages souriants, accueillants, tu te gênes pas quand tu viens, tu es à l'aise quand tu viens, et tu sens la présence du saint Esprit, je sens ça sur moi. » (Amélie, entretien du 19 juin 2001, Tahiti).

40. Ce dialogue entre un converti pentecôtiste et son frère, diacre de l'Église protestante traditionnelle, illustre bien l'écart entre la logique culturelle d'honneur et l'évangile relationnel des assemblées de Dieu : « Dans ma famille, les frères et sœurs ne se parlent plus, même dans leur religion, ils ne se parlent plus entre eux. (...) J'ai dit : "pourquoi, tu es diacre, pourquoi tu veux pas te soumettre, aller un peu en dessous des autres ?" Lui, il dit: "Je peux pas aller en dessous d'eux". C'est comme ça que j'ai pensé il connaît pas Jésus. » (Vincent, entretien du 19 juin 2001).

41. Assemblée de Dieu de Papeete, entretien du 14 avril 2001.

42. Nicolas et Lena Venditti, op. cit., p. 279.

43. Pasteur Amaru, séminaire de couple à Raiatea (îles Sous-le-Vent), le 30 juin 2001.

44. Éric Neveu, Une société de communication?, Paris, Montchrétien, 2001. Les professionnels du travail symbolique qui agissent en tant que « montreurs de communication » partagent « souvent ce qui est inséparablement une croyance profonde et un intérêt statutaire : faire de la communication une injonction, une pratique nécessaire pour contribuer à une société plus harmonieuse» (p. 110 ss.).

45. Ibid.

46. Walter J. HOLLENWEGER, op. cit., p. 466. La dernière phrase (entre guillemets) est une citation de Christian Lalive d'Épinay, Haven of the Masses. A Study of the Pentecostal Movement in Chile, Londres, Lutterworth, 1969, p. 53.

47. Jacques Nicole, Au pied de l'écriture, histoire de la traduction de la Bible en tahitien, Haere po no Tahiti, 1988, p. 276.

48. Pasteur Levant, culte du 27 mars 2001 à Papeete (Tahiti).

49. Yves Winkin, 2001, Anthropologie de la communication. De la théorie au terrain, Paris, De Boeck Université/Seuil, p. 60.

50. Walter J. HOLLENWEGER, op. cit., p. 466.

51. Jean-Paul Willaime, op. cit., p. 11.

52. Culte à l'assemblée de Dieu de Taravao (presqu'île de Tahiti), 22 juillet 2001. 


\section{RÉSUMÉS}

En s'en tenant à une lecture de l'émotion en religion comme expérience "élémentaire " présociale, la sociologie ne peut rendre compte de toutes les dimensions du pentecôtisme contemporain. L'analyse des Assemblées de Dieu de Polynésie française met à jour non un simple processus régressif, mais une articulation complexe entre personnalisation des croyances, travail institutionnel et expressions émotionnelles. Au-delà de l'effervescence apparemment désordonnée des cultes, celles-ci s'inscrivent finalement dans le cadre d'un "évangile relationnel » en affinité avec l'idéologie moderne de la « société de communication ».

Sociology can not understand all the aspects of contemporary Pentecostalism when it confines the reading of religious emotion as pre-social "elementary" experience. The analysis of the Assemblies of God of French Polynesia shows a complex combination between personalization of beliefs, institutional work and emotional expressions rather than a plain backward process. Beyond the seemingly disorganized effervescence of the worship, these emotional expressions are part of a "Relationship Gospel" in affinity to the modern ideology of the "Society of Communication".

Al tenerse a una lectura de la emoción en religión como experiencia "elementaria" pre-social, la sociologica no puede dar cuenta de todas las dimensiones del pentecotismo contemporáneo. El análisis de las Assembleas de Dios en Polinesia francesca revela no un sencillo proceso regresivo sino una articulation compleja entre personalización de las creyencias, trabajo institucional y expresiones emocionales. Aquellas, más allá de la efervescencia al parecer desordenado de los cultos, se inscriben en el marco de un "evangelio relacional" en afinidad con la ideología moderna de la "sociedad de comunicaciones".

\section{INDEX}

Mots-clés : Assemblées de Dieu, emotion en religion, pentecôtisme, Polynésie

\section{AUTEUR}

YANNICK FER

Centre d'Études Interdisciplinaires des Faits Religieux - EHESS 